\title{
Investigation of Transfer Characteristics of Complex Surfaces by using Periodic Models
}

\author{
Gergely Kristóf \\ Associate professor, Department of Fluid Mechanics, Faculty of Mechanical Engineering, \\ Budapest University of Technology and Economics \\ kristof@ara.bme.hu
}

\begin{abstract}
Application of periodic CFD models greatly simplifies the evaluation of transfer characteristics of complex surfaces, moreover, facilitates the application of scale resolving turbulence models such as large eddy simulation. Owing to the substantial reduction of simulation domain size, and reduction of computing time of individual cases, periodic models are favorable for geometrical optimization. Geometrical simplicity also helps researchers in finding general tendencies as well as generalizing the model results. Practical applications are demonstrated in A) development of heat exchanger tubes with internal grooving and B) improving ventilation characteristics of the urban complex by optimizing building configurations. Possible technical realizations, benefits from the reduction of modelling uncertainties, as well as the limits of applicability are discussed.
\end{abstract}

